\title{
Sobolev's inequality for Riesz potentials of functions in generalized Morrey spaces with variable exponent attaining the value 1 over non-doubling measure spaces
}

\author{
Yoshihiro Sawano ${ }^{1,2^{*}}$ and Tetsu Shimomura ${ }^{3}$
}

\author{
"Correspondence: \\ ysawano@tmu.ac.jp \\ ${ }^{1}$ Department of Mathematics, Kyoto \\ University, Kyoto, 606-8502, Japan \\ ${ }^{2}$ Current address: Department of \\ Mathematics and Information \\ Sciences, Tokyo Metropolitan \\ University, Minami-Ohsawa 1-1, \\ Hachioji-shi, Tokyo 192-0397, Japan \\ Full list of author information is \\ available at the end of the article
}

\begin{abstract}
Our aim in this paper is to give Sobolev's inequality for Riesz potentials of functions in generalized Morrey spaces with variable exponent attaining the value 1 over non-doubling measure spaces. The main result is oriented to the outrange of the well-known Adams theorem.

MSC: Primary 31B15; secondary 46E35; 26A33
\end{abstract}

Keywords: Riesz potentials; Sobolev's inequality; Morrey spaces of variable exponents; non-doubling measure

\section{Introduction}

The boundedness of fractional integral operators on Morrey spaces is known as the Adams theorem. Recently, many endpoint results have been obtained for this theorem, and in this paper we extend them to generalized Morrey spaces with variable exponent attaining the value 1 over non-doubling measure spaces.

In 1938 Morrey observed that a weaker regularity sufficed in order that the solutions in elliptic differential equations were smooth [1]. This observation grew up to be a useful tool for partial differential equations in general. Nowadays, his technique turned out to be a wide theory of function spaces called Morrey spaces; see also [2]. The (original) Morrey space $L^{p, \lambda}\left(\mathbf{R}^{N}\right)$ with $1 \leq p<\infty$ and $0<\lambda \leq N$ is a normed space whose norm is given by $\|f\|_{L^{p, \lambda}} \equiv \sup _{x \in \mathbf{R}^{N}, r>0}\left(\frac{1}{r^{N-\lambda}} \int_{B(x, r)}|f(y)|^{p} d y\right)^{\frac{1}{p}}$ for $f \in L_{\mathrm{loc}}^{p}\left(\mathbf{R}^{N}\right)$.

We are oriented to building up a theory of a metric measure space $(X, d, \mu)$ for which the notion of dimension is not equipped with $\mu$, where $d$ is a distance function and $\mu$ is a Borel measure. For example, we encounter the situation where more than one dimension comes into play.

1. In $\mathbb{R}^{3}$ consider the set $X \equiv Y \cup Z$, where $Y \equiv\{(x, 0,0): x \in \mathbb{R}\}$ and $Z \equiv\left\{(x, y, z): z=\sqrt{x^{2}+y^{2}}\right\}$. Denote by $\mathcal{H}^{s}$ the $s$-dimensional Hausdorff measure in $\mathbb{R}^{3}$ for $0 \leq s \leq 3$. Consider $\mu \equiv \mathcal{H}^{1}\left|Y+\mathcal{H}^{2}\right| Z$. Then $\mu$ has two different dimensions.

2. The above example is a little artificial. Consider the Cantor dust, which is given by $E \equiv \bigcap_{j=1}^{\infty} E_{j}$, where $E_{j}$ is given recursively by

$$
E_{0} \equiv[0,1]^{2}
$$

\section{Springer}

(C) 2013 Sawano and Shimomura; licensee Springer. This is an Open Access article distributed under the terms of the Creative Commons Attribution License (http://creativecommons.org/licenses/by/2.0), which permits unrestricted use, distribution, and reproduction in any medium, provided the original work is properly cited. 


$$
E_{j+1}=\bigcup_{e \in\{0,1\}^{2}}\left(\frac{1}{2} e+\frac{1}{4} E_{j}\right)=\left\{\frac{1}{2} e+\frac{1}{4} x: x \in E_{j}, e \in\{0,1\}^{2}\right\} \subset \mathbb{R}^{2} .
$$

Then the Hausdorff dimension of $E$ is 1 while each $E_{j}$ is a union of closed cubes in $\mathbb{R}^{2}$. As the second example shows, when we consider the subsets in $\mathbb{R}^{2}$, it is natural to approximate them with sets having different dimension. Faced with such an inconvenience, we work on a separable metric space $X$ equipped with a nonnegative Radon measure $\mu$, where the notion of dimensions of $X$ and $\mu$ does not come into play.

We assume that

$$
\mu(\{x\})=0
$$

for all $x \in X$. By $B(x, r)$ we denote the open ball centered at $x$ of radius $r>0$. We assume

$$
0<\mu(B(x, r))<\infty
$$

for $x \in X$ and $r>0$ for simplicity. We write $d(x, y)$ for the distance of the points $x$ and $y$ in $X$. We are interested in the operator of the form $f \mapsto \int_{X} K(\cdot, y) f(y) d \mu(y)$, where $f$ is a $\mu$-measurable function with a certain $\mu$-integrability. Note that (1.1) is indispensable because we envisage examples in which $K: X \times X \rightarrow[0, \infty]$ has singularity at the diagonal. Meanwhile, (1.2) does not lose any generality; it is just a matter of restricting $X$ to $\operatorname{supp}(\mu)$

Let $G$ be a bounded open set in $X$. Our argument to follow heavily depends upon its diameter $d_{G}$. Let $p \geq 1, v>0$ and $k>0$. Define the Morrey norm $\|f\|_{L^{p, v, \beta ; k}(G)}$ by

$$
\|f\|_{L^{p, \nu, \beta ; k}(G)} \equiv \sup _{x, r}\left(\frac{r^{\nu}(\log (e+1 / r))^{\beta}}{\mu(B(x, k r))} \int_{B(x, r) \cap G}|f(y)|^{p} d \mu(y)\right)^{1 / p}
$$

for $\mu$-measurable functions $f$, where $(x, r)$ runs over all elements in $G \times\left(0, d_{G}\right)$. The Morrey space $L^{p, v, \beta ; k}(G)$ is the set of all $\mu$-measurable functions $f$ for which the norm $\|f\|_{L^{p, v, \beta ; k}(G)}$ is finite. When $\beta=0, L^{p, v, \beta ; k}(G)$ is denoted by $L^{p, v ; k}(G)$.

In this paper, we aim to understand how the fractional integral operators behave in generalized Morrey spaces with variable exponent attaining the value 1 over non-doubling measure spaces. We obtain the following constant exponent case as a corollary of a more general theorem in non-doubling and variable exponent setting (see Theorem 2.1 below). The result is new even for a constant exponent case.

Theorem 1.1 Let $\alpha, \beta, v$ be constants. Define an index $p^{*}$ by $\frac{1}{p^{*}}=1-\frac{\alpha}{v}$, and let $\gamma>1$. Define

$$
U_{\alpha, 9} f(x) \equiv \int_{G} \frac{d(x, y)^{\alpha}}{\mu(B(x, 9 d(x, y)))} f(y) d \mu(y)
$$

for a positive $\mu$-measurable function $f$. Then there exists a constant $C>0$ such that

$$
\begin{aligned}
& \frac{1}{\mu(B(z, 4 r))} \int_{B(z, r)} U_{\alpha, 9} f(x)^{p^{*}}\left(\log \left(e+U_{\alpha, 9} f(x)\right)\right)^{-\gamma+\alpha \beta p^{*} / v} d \mu(x) \\
& \leq C r^{-v}(\log (e+1 / r))^{-\beta-\gamma+1}
\end{aligned}
$$


for all $z \in G$ and $0<r<d_{G}$, whenever $f$ is a nonnegative $\mu$-measurable function on $G$ satisfying $\|f\|_{L^{1, v, \beta ; 2}(G)} \leq 1$.

We remark that $U_{\alpha, 9}$ extends naturally the fractional integral operator defined by Kokilashvili on $\mathbb{R}^{N}$ [3]. We also remark that an extension to quasi-metric spaces was performed in [4].

Theorem 1.1 describes the missing part of the Adams inequality, which we recall now.

Proposition 1.2 (Adams, [5]) Let $0<\alpha<n, 1<p, q<\infty$ and $0<\lambda \leq N$. Assume $\frac{1}{q}=\frac{1}{p}-\frac{\alpha}{\lambda}$. Then there exists a constant $C>0$ such that $\left\|I_{\alpha} f\right\|_{L^{q, \lambda}} \leq C\|f\|_{L^{p, \lambda}}$ for all $f \in L^{p, \lambda}\left(\mathbf{R}^{N}\right)$.

In the endpoint case, when $p=1$, two natural questions about Proposition 1.2 arise.

1. Can we prove a similar result by enlarging $L^{q, \lambda}\left(\mathbf{R}^{N}\right)$ slightly?

2. Can we prove a similar result by shrinking $L^{p, \lambda}\left(\mathbf{R}^{N}\right)$ slightly?

The second question is considered in [6]. We aim to consider the first question in the present paper.

It is the condition on $\mu$ that counts in the present paper; we do not postulate on $\mu$ the so-called doubling condition. Recall that a Radon measure $\mu$ is said to be doubling if there exists a constant $C>0$ such that $\mu(B(x, 2 r)) \leq C \mu(B(x, r))$ for all $x \in \operatorname{supp}(\mu)(=X)$ and $r>0$. Otherwise, $\mu$ is said to be non-doubling. In connection with the $5 r$-covering lemma, the doubling condition had been a key condition in harmonic analysis. However, Nazarov, Treil and Volberg showed that the doubling condition was not necessary by using the modified maximal operator [7, 8]. The idea of replacing $d(x, y)$ with $9 d(x, y)$ in $(1.3)$ originates from these papers. In the present paper, we show that this idea works even for Riesz potentials.

Here, we summarize the structure of the space $L^{p, v ; k}(G)$ in Proposition 1.3 and Remark 1.4 below. Note that the Morrey space $L^{p, v ; k}(G)$ does depend upon the parameter $k$, which is illustrated by the following proposition.

Proposition 1.3 ([9]) There does exist a bounded separable metric space $(X, d, \mu)$ such that $L^{p, v ; 2}(G)$ and $L^{p, v ; 4}(G)$ do not coincide as sets.

About the modified Morrey norm, we have the following remarks.

Remark 1.4 Let $f: X \rightarrow[0, \infty]$ be a $\mu$-measurable function and $G$ be a bounded open subset of $X$.

1. From the definition of the norms, we learn $\|f\|_{L^{p, v ; k_{2}(G)}} \leq\|f\|_{L^{p, v ; k_{1}(G)}}$ for all $k_{2}>k_{1}>0$ and $p \geq 1$.

2. If $p_{2} \geq p_{1} \geq 1, k \geq 1$ and $v_{1} / p_{1}=v_{2} / p_{2}>0$, then $\|f\|_{L^{p_{1}, v_{1} ; k}(G)} \leq\|f\|_{L^{p_{2}, v_{2} ; k}(G)}$ by the Hölder inequality.

3. If $\mu$ is a doubling measure, then $\|f\|_{L^{p, v ; k}(G)}$ and $\|f\|_{L^{p, v ; 1}(G)}$ are equivalent for all $p \geq 1$, $k>0$ and $v>0$.

Our result can be readily translated into the Morrey space $L^{p, \lambda}(G)$, where $L^{p, \lambda}(G)$ is the set of all functions $f$ such that

$$
\|f\|_{L^{p, \lambda}(G)} \equiv \sup _{x \in G, r \in\left(0, d_{G}\right)}\left(\frac{1}{r^{N-\lambda}} \int_{G \cap B(x, r)}|f(y)|^{p} d y\right)^{1 / p} .
$$


Keeping our fundamental result, Theorem 1.1, in mind, let us describe our results in full generality. We seek to establish Sobolev's inequality for Riesz potentials of functions in generalized Morrey spaces with variable exponent attaining the value 1 over non-doubling measure spaces, which will extend the results in our earlier papers [10-17]. In the present paper, we show that a modification enables us to obtain boundedness results in generalized Morrey spaces with variable exponent attaining the value 1 over non-doubling measure spaces even when

$$
\inf _{x \in X} p(x)=1
$$

We consider variable exponents $p(\cdot)$ and $q(\cdot)$ on $X$ such that

(P1) $1 \leq p_{-} \equiv \inf _{x \in X} p(x) \leq \sup _{x \in X} p(x) \equiv p_{+}<\infty$;

(P2) $|p(x)-p(y)| \leq C / \log \left(e+d(x, y)^{-1}\right)$ whenever $x \in X$ and $y \in X$;

(Q1) $-\infty<q_{-} \equiv \inf _{x \in X} q(x) \leq \sup _{x \in X} q(x) \equiv q_{+}<\infty$;

(Q2) $|q(x)-q(y)| \leq C / \log \left(e+\left(\log \left(e+d(x, y)^{-1}\right)\right)\right)$ whenever $x \in X$ and $y \in X$.

In general, if $p(\cdot)$ satisfies (P2) (resp. $q(\cdot)$ satisfies $(\mathrm{Q} 2))$, then $p(\cdot)($ resp. $q(\cdot))$ is said to satisfy the log-Hölder (resp. loglog-Hölder) condition. Observe that these conditions are much weaker than Lipschitz continuity.

Next, we redefine the Riesz potential. Recall that we are working on a bounded subset $G$ of $X$. For a bounded $\mu$-measurable function $\alpha(\cdot): X \rightarrow(0, \infty)$ and a constant $\kappa>0$, we define the Riesz potential of (variable) order $\alpha(\cdot)$ for a nonnegative $\mu$-measurable function $f$ on $G$ by

$$
U_{\alpha(\cdot), k} f(x) \equiv \int_{G} \frac{d(x, y)^{\alpha(x)} f(y)}{\mu(B(x, \kappa d(x, y)))} d \mu(y) .
$$

Here and in what follows, we tacitly assume that $f=0$ outside $G$. Observe that this naturally extends the Riesz potential operator

$$
U_{\alpha} f(x) \equiv \int_{\mathbf{R}^{N}} \frac{f(y)}{|x-y|^{N-\alpha}} d y
$$

when $(X,|\cdot|, \mu)$ is the $N$-dimensional Euclidean space and $\mu=d x$.

We also assume

$$
\alpha_{-} \equiv \inf _{x \in X} \alpha(x)>0
$$

for $\alpha(\cdot)$ appearing in the definition of the operator $U_{\alpha(\cdot), \kappa}$.

Now, we are going to formulate our results in full generality. First of all, we set

$$
\Phi(x, r)=\Phi_{p(\cdot), q(\cdot)}(x, r) \equiv r^{p(x)}(\log (c+r))^{q(x)} \quad(x \in X, r>0) ;
$$

here we assume

$(\Phi) \Phi_{p(\cdot), q(\cdot)}(x, \cdot)$ is convex on $[0, \infty)$ for every $x \in X$.

Note that $(\Phi)$ holds for some $c \geq e$ if and only if there is a positive constant $K$ such that

$$
K(p(x)-1)+q(x) \geq 0 \quad \text { for all } x \in X
$$


(see [18, Theorem 5.1]). Observe from $(\Phi)$ that the function $t \mapsto t^{-1} \Phi(x, t)$ is nondecreasing on $(0, \infty)$ for fixed $x \in X$.

Now, we redefine and extend the definition of Morrey norms adapted to the function $\Phi$. Let $k>1$ be a fixed parameter and let $G$ be a bounded subset of $X$. Let us denote by $d_{G}$ the diameter of $G$. For bounded $\mu$-measurable functions $v: X \rightarrow(0, \infty)$ and $\beta: X \rightarrow(-\infty, \infty)$, we introduce the family $L^{\Phi, v, \beta ; k}(G)$ of all $\mu$-measurable functions $f$ on $G$ such that for some $\lambda \in(0, \infty)$,

$$
\sup _{x \in G, 0<r<d_{G}} \frac{r^{\nu(x)}(\log (c+1 / r))^{\beta(x)}}{\mu(B(x, k r))} \int_{G \cap B(x, r)} \Phi(y,|f(y)| / \lambda) d \mu(y) \leq 1 .
$$

Denote by $\|f\|_{L^{\Phi, v, \beta ; k}(G)}$ the smallest value of $\lambda$ satisfying (1.6). Note that $v$ and $\beta$ need not be continuous.

The space $L^{\Phi, v, \beta ; k}(G)$ is a further generalization of generalized Morrey spaces with nondoubling measures on $\mathbf{R}^{N}$, which are taken up in $[19,20]$. Nowadays, generalized Morrey spaces are not generalization for its own sake. Note that generalized Morrey spaces occur naturally when we consider the limiting case as Proposition 1.5 below shows.

Proposition 1.5 ([21, Theorem 5.1]) Let $1<p<\infty$ and $0<\lambda<N$. Then there exists a positive constant $C_{p, \lambda}$ such that

$$
\int_{B}|f(x)| d x \leq C_{p, \lambda}|B|(1+|B|)^{-\frac{1}{p}} \log \left(e+\frac{1}{|B|}\right)\left\|(1-\Delta)^{\lambda / 2 p} f\right\|_{L^{p, \lambda}}
$$

holds for all $f \in L^{p, \lambda}\left(\mathbf{R}^{N}\right)$ with $(1-\Delta)^{\lambda / 2 p} f \in L^{p, \lambda}\left(\mathbf{R}^{N}\right)$ and for all balls $B$.

In view of the integral kernel of $(1-\Delta)^{-\alpha / 2}$ (see [22]) and the Adams theorem, we have

$$
(1-\Delta)^{-\alpha / 2}: L^{p, \lambda}\left(\mathbf{R}^{N}\right) \rightarrow L^{q, \lambda}\left(\mathbf{R}^{N}\right)
$$

is bounded as long as

$$
1<p, q<\infty, \quad 0<\lambda \leq N, \quad \frac{1}{q}=\frac{1}{p}-\frac{\alpha}{\lambda} .
$$

However, if $\alpha=\frac{\lambda}{p}$, the number $q$ not being finite, the boundedness assertion (1.7) is no longer true. Hence, Proposition 1.5 can be considered as a substitute of (1.7). Proposition 1.5 shows that the price to pay is the factor $\log \left(e+\frac{1}{|Q|}\right)$. We refer to [21] for a counterexample showing that (1.7) is no longer true for $\alpha=\frac{\lambda}{p}$.

Remark that if $X=\mathbf{R}^{N}$, the parameter $k$ is not essential as long as $k>1$ as Proposition 1.6 below shows.

Proposition 1.6 ([9]) Let $k_{1}, k_{2}>1$ and $X=\mathbf{R}^{N}$ be the Euclidean space. Suppose that $G$ is a bounded open set. Assume in addition that $v$ and $\beta$ satisfy the log-Hölder continuity $(\mathrm{P} 2)$ and the loglog-Hölder continuity $(\mathrm{Q} 2)$, respectively. Then the spaces $L^{\Phi, v, \beta ; k_{1}}(G)$ and $L^{\Phi, v, \beta ; k_{2}}(G)$ coincide as sets and their norms are equivalent. 
What is different from the classical setting endowed with Lebesgue measure is that we need to take an attentive care of the parameters $k$ appearing in (1.6). For example, unlike the doubling measure spaces, we need delicate geometric observations (see (2.19) for example).

Among many other function spaces, Morrey spaces with variable exponents are worth investigating because our result Theorem 2.1 shows that the smoothing effect that the fractional integral operators enjoy is local. It is believed that $p$ in the definition of $L^{p, v ; k}(G)$ measures the local integrability, while $v$ seems to control the global integrability.

Here we make a historical remark about the research of this field. The case $\mu=d x$ of Theorem 2.1 is covered in [12, Theorem 1.2] and [13, Corollary 4.2]. Roughly speaking, we seek to discuss the boundedness of the operator $U_{\alpha(\cdot), 9}: f \mapsto U_{\alpha(\cdot), 9} f$ from the Morrey space $L^{\Phi, v, \beta ; 2}(G)$ to another Morrey space $L^{\Psi, v, \beta ; 2}(G)$ with suitable $\Psi(x, r)$, which extends the results in [10-17]. When $p_{-}>1$, the maximal functions play a crucial tool by Hedberg's trick (see [23]). In view of $[7,8,24,25]$, the modified maximal operator is bounded and this was useful for the case when $p_{-}>1$. In case $p_{-}=1$, our strategy is to give an estimate of $U_{\alpha(\cdot), 9} f$ by the use of another Riesz-type potentials of order 0 , which plays a role of the maximal functions. For related results, see [26-28].

Finally, we explain some notations used in the present paper. The function $\chi_{E}$ denotes the characteristic function of $E$. Throughout the present paper, let $C$ denote various constants independent of the variables in question. In analogy with $p_{ \pm}$and $q_{ \pm}$, we define $\alpha_{ \pm}$, $\beta_{ \pm}$and $\nu_{ \pm}$.

\section{Sobolev's inequality in the case $p_{-}=1$}

Recall that $\alpha: X \rightarrow(0, \infty), v: X \rightarrow(0, \infty)$ and $\beta: X \rightarrow(-\infty, \infty)$ are bounded $\mu$ measurable functions and $\alpha_{-}>0$. Throughout this section, we additionally assume that

$$
\inf _{x \in X}(1 / p(x)-\alpha(x) / v(x))>0
$$

Note that $v(x) \geq \alpha(x) p(x)$ for $x \in X$. Thus, from $p_{-} \geq 1$ and (1.4), we have

$$
\nu_{-} \geq \alpha_{-}>0 .
$$

Our first aim is to give the following Morrey version of Sobolev-type inequality for Riesz potentials

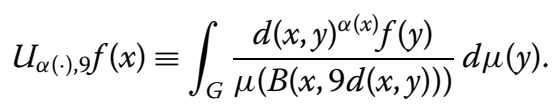

We consider the Sobolev exponent

$$
1 / p^{*}(x) \equiv 1 / p(x)-\alpha(x) / v(x) \quad(x \in X)
$$

and the new modular function

$$
\Psi(x, t) \equiv t^{p^{\prime \prime}(x)}(\log (c+t))^{p^{*}(x)(q(x) / p(x)+\alpha(x) \beta(x) / v(x))} \quad(x \in X, t \in(0, \infty)) .
$$


Theorem 2.1 Assume that $p(\cdot)$ satisfies (P1) with $p_{-}=1$, (P2) and (2.1). Then, for each $\varepsilon>0$, there exists a constant $C>0$ such that

$$
\begin{aligned}
& \frac{1}{\mu(B(z, 4 r))} \int_{B(z, r)} \Psi\left(x, U_{\alpha(\cdot), 9} f(x)\right)\left(\log \left(c+U_{\alpha(\cdot), 9} f(x)\right)\right)^{-(1+\varepsilon)} d x \\
& \quad \leq C r^{-v(z)}(\log (c+1 / r))^{-\beta(z)-\varepsilon}
\end{aligned}
$$

for all $z \in G$ and $0<r<d_{G}$, whenever $f$ is a nonnegative $\mu$-measurable function on $G$ satisfying $\|f\|_{L^{\Phi, v, \beta ; 2}(G)} \leq 1$.

Remark 2.2 In Theorem 2.1, it is known that we cannot take $\varepsilon=0$ (see [14, Remark 3.3] and O'Neil [28, Theorem 5.2]).

Here we outline the proof of Theorem 2.1.

For $\varepsilon>0$ and $x \in X$, and setting

$$
\rho_{\varepsilon}(r) \equiv \frac{1}{\mu(B(x, 9 r))}(\log (c+1 / r))^{-\varepsilon-1}
$$

we consider the logarithmic potential (of order 0 )

$$
J_{\varepsilon} f(x)=\int_{G} \rho_{\varepsilon}(d(x, y))|f(y)|^{p(y)}(\log (c+|f(y)|))^{q(y)} d \mu(y) .
$$

Decompose

$$
\begin{aligned}
U_{\alpha(\cdot), 9} f(x) & =\int_{B(x, \delta)} \frac{d(x, y)^{\alpha(x)} f(y)}{\mu(B(x, 9 d(x, y)))} d \mu(y)+\int_{G \backslash B(x, \delta)} \frac{d(x, y)^{\alpha(x)} f(y)}{\mu(B(x, 9 d(x, y)))} d \mu(y) \\
& =I_{1}(\delta)+I_{2}(\delta), \quad \text { say. }
\end{aligned}
$$

Following the Hedberg trick [23], we plan to control $I_{1}(\delta)$ by $J_{\varepsilon} f(x)$ not by maximal functions (Lemma 2.3). Next, we estimate $I_{2}(\delta)$ by the use of Young's inequality (Lemma 2.4). Finally, an optimization (see (2.22) below), together with the boundedness property of $J_{\varepsilon}$ (Lemma 2.5), yields the desired estimate $U_{\alpha(\cdot), 9} f(x)$ in terms of $J_{\varepsilon} f(x)$.

Lemma 2.3 For $0<\delta<d_{G}, x \in G$ and a nonnegative $\mu$-measurable function $f$ on $G$, set

$$
I_{1}(\delta) \equiv \int_{B(x, \delta)} \frac{d(x, y)^{\alpha(x)} f(y)}{\mu(B(x, 9 d(x, y)))} d \mu(y)
$$

Let $\varepsilon>0$ be fixed. Then there exists a constant $C>0$ such that

$$
\begin{aligned}
I_{1}(\delta) \leq & C\left\{\delta^{\alpha(x)-v(x) / p(x)}\left(\log \left(c+\delta^{-1}\right)\right)^{-(q(x)+\beta(x)) / p(x)}\right. \\
& \left.+\delta^{\alpha(x)+(p(x)-1) v(x) / p(x)}\left(\log \left(c+\delta^{-1}\right)\right)^{\beta(x)-(q(x)+\beta(x)) / p(x)+(1+\varepsilon)} J_{\varepsilon} f(x)\right\},
\end{aligned}
$$

whenever $f$ is a nonnegative $\mu$-measurable function on G satisfying $\|f\|_{L^{\Phi, v, \beta ; 2}(G)} \leq 1$. 
Proof Recall that $\mu$ does not charge a point $x$ (see (1.1) above) and that $\alpha_{-}>0$ by virtue of (2.2). We thus have

$$
\begin{aligned}
& \int_{B(x, \delta)} \frac{d(x, y)^{\alpha(x)}}{\mu(B(x, 9 d(x, y)))} d \mu(y) \\
& \quad=\sum_{j=1}^{\infty} \int_{B\left(x, 2^{-j+1} \delta\right) \backslash B\left(x, 2^{-j} \delta\right)} \frac{d(x, y)^{\alpha(x)}}{\mu(B(x, 9 d(x, y)))} d \mu(y) \\
& \quad \leq \sum_{j=1}^{\infty} \int_{B\left(x, 2^{-j+1} \delta\right)} \frac{\left(2^{-j+1} \delta\right)^{\alpha(x)}}{\mu\left(B\left(x, 2^{-j+3} \delta\right)\right)} d \mu(y) \\
& \quad \leq \sum_{j=1}^{\infty}\left(2^{-j+1} \delta\right)^{\alpha(x)}=\frac{1}{1-2^{-\alpha(x)}} \delta^{\alpha(x)} \leq \frac{1}{1-2^{-\alpha_{-}}} \delta^{\alpha(x)}=C \delta^{\alpha(x)} .
\end{aligned}
$$

Consequently,

$$
\int_{B(x, \delta)} \frac{d(x, y)^{\alpha(x)}}{\mu(B(x, 9 d(x, y)))} d \mu(y) \leq C \delta^{\alpha(x)} .
$$

From ( $\Phi)$, observe that the function $t \mapsto t^{-1} \Phi(x, t)$ is nondecreasing on $(0, \infty)$ for fixed $x \in X$. With this in mind, for $k>0$, which we specify in (2.10), we decompose and estimate the integral defining $I_{1}(\delta)$ according to the level set $\{|f|>k\}$ :

$$
\begin{aligned}
I_{1}(\delta) \leq & k \int_{B(x, \delta)} \frac{d(x, y)^{\alpha(x)}}{\mu(B(x, 9 d(x, y)))} d \mu(y) \\
& +\int_{B(x, \delta)} \frac{d(x, y)^{\alpha(x)}}{\mu(B(x, 9 d(x, y)))} f(y)\left(\frac{f(y)}{k}\right)^{p(y)-1}\left(\frac{\log (c+f(y))}{\log (c+k)}\right)^{q(y)} d \mu(y) .
\end{aligned}
$$

The first term is estimated by (2.9):

$$
k \int_{B(x, \delta)} \frac{d(x, y)^{\alpha(x)}}{\mu(B(x, 9 d(x, y)))} d \mu(y) \leq C k \delta^{\alpha(x)} .
$$

Recall also that $\rho_{\varepsilon}$ is defined by (2.6). Inserting them, we have

$$
\begin{aligned}
I_{1}(\delta) \leq & C\left\{k \delta^{\alpha(x)}+\int_{B(x, \delta)} \frac{d(x, y)^{\alpha(x)}}{\mu(B(x, 9 d(x, y)))} g(y)\left(\frac{1}{k}\right)^{p(y)-1}\left(\frac{1}{\log (c+k)}\right)^{q(y)} d \mu(y)\right\} \\
\leq & C\left\{k \delta^{\alpha(x)}+\delta^{\alpha(x)}\left(\log \left(c+\delta^{-1}\right)\right)^{1+\varepsilon}\right. \\
& \left.\times \int_{B(x, \delta)} \rho_{\varepsilon}(d(x, y)) g(y) k^{1-p(y)}\left(\frac{1}{\log (c+k)}\right)^{q(y)} d \mu(y)\right\},
\end{aligned}
$$

where $g(y)=f(y)^{p(y)}(\log (c+f(y)))^{q(y)}$. We set

$$
k \equiv k(x)=\delta^{-v(x) / p(x)}\left(\log \left(c+\delta^{-1}\right)\right)^{-(q(x)+\beta(x)) / p(x)} .
$$

For $y \in B(x, \delta)$, from (P2) and the boundedness of $\log p, q, \beta$ and $v$, we deduce

$$
|(p(x)-p(y)) \log k| \leq C
$$


so that

$$
k^{-p(y)} \leq C k^{-p(x)} .
$$

Similarly, by (Q2) we have

$$
(\log (c+k))^{-q(y)} \leq C(\log (c+k))^{-q(x)} .
$$

Consequently, it follows from (2.11) and (2.12) that

$$
\begin{aligned}
I_{1}(\delta) \leq & C\left\{k \delta^{\alpha(x)}\right. \\
& \left.+\delta^{\alpha(x)}\left(\log \left(c+\delta^{-1}\right)\right)^{1+\varepsilon} k^{1-p(x)}\left(\frac{1}{\log (c+k)}\right)^{q(x)} \int_{B(x, \delta)} \rho_{\varepsilon}(d(x, y)) g(y) d \mu(y)\right\} .
\end{aligned}
$$

Recall now that $J_{\varepsilon} f(x)$ is given by (2.7). Observe also that

$$
\log (c+k)=\log \left(c+\delta^{-v(x) / p(x)}\left(\log \left(c+\delta^{-1}\right)\right)^{-(q(x)+\beta(x)) / p(x)}\right) \sim \log \left(c+\delta^{-1}\right),
$$

since $v_{-}>0, p_{-} \geq 1$ and $p, q, \beta$ are all bounded. Thus,

$$
\begin{aligned}
I_{1}(\delta) \leq & C\left\{\delta^{\alpha(x)-v(x) / p(x)}\left(\log \left(c+\delta^{-1}\right)\right)^{-(q(x)+\beta(x)) / p(x)}\right. \\
& \left.+\delta^{\alpha(x)}\left(\log \left(c+\delta^{-1}\right)\right)^{1+\varepsilon-q(x)}\left(\delta^{\nu(x) / p(x)}\left(\log \left(c+\delta^{-1}\right)\right)^{(q(x)+\beta(x)) / p(x)}\right)^{p(x)-1} J_{\varepsilon} f(x)\right\} \\
\leq & C\left\{\delta^{\alpha(x)-v(x) / p(x)}\left(\log \left(c+\delta^{-1}\right)\right)^{-(q(x)+\beta(x)) / p(x)}\right. \\
& \left.+\delta^{\alpha(x)+(p(x)-1) v(x) / p(x)}\left(\log \left(c+\delta^{-1}\right)\right)^{\beta(x)-(q(x)+\beta(x)) / p(x)+(1+\varepsilon)} J_{\varepsilon} f(x)\right\} .
\end{aligned}
$$

Now, the result follows.

In our earlier work, we obtained the following lemma, whose proof is simple; we do not recall the proof.

The quantity $I_{2}(\delta)$ will be taken care of by using the next lemma.

Lemma 2.4 ([9, Lemma 5.4]) Let $f$ be a nonnegative $\mu$-measurable function on $G$ such that

$$
\|f\|_{L^{\Phi, v, \beta ; 2}(G)} \leq 1
$$

Then

$$
\begin{aligned}
I_{2}(\delta) & \equiv \int_{G \backslash B(x, \delta)} \frac{d(x, y)^{\alpha(x)} f(y)}{\mu(B(x, 9 d(x, y)))} d \mu(y) \\
& \leq C \delta^{\alpha(x)-v(x) / p(x)}\left(\log \left(c+\delta^{-1}\right)\right)^{-(q(x)+\beta(x)) / p(x)}
\end{aligned}
$$

for $x \in G$ and $0<\delta<d_{G}$. 
What remains to show for the proof of Theorem 2.1 is to give a boundedness property on Morrey spaces for $J_{\varepsilon} f(x)$.

Lemma 2.5 There exists a constant $C>0$ such that

$$
\frac{1}{\mu(B(z, 4 r))} \int_{B(z, r)} J_{\varepsilon} f(x) d \mu(x) \leq C r^{-v(z)}(\log (c+1 / r))^{-\beta(z)-\varepsilon}
$$

$\forall z \in G, 0<r<d_{G}$ and nonnegative $\mu$-measurable functions $f$ satisfying $\|f\|_{L^{\Phi, v, \beta ; 2}(G)} \leq 1$.

Proof For $z \in G$ and $0<r<d_{G}$, write

$$
\begin{aligned}
J_{\varepsilon} f(x) & =\int_{B(z, 2 r)} \rho_{\varepsilon}(d(x, y)) g(y) d \mu(y)+\int_{G \backslash B(z, 2 r)} \rho_{\varepsilon}(d(x, y)) g(y) d \mu(y) \\
& =J_{1}(x)+J_{2}(x),
\end{aligned}
$$

where $g(y)=f(y)^{p(y)}(\log (c+f(y)))^{q(y)}$. Then we have

$$
\begin{aligned}
\int_{B(z, r)} J_{1}(x) d \mu(x) & =\int_{B(z, 2 r)}\left(\int_{B(z, r)} \rho_{\varepsilon}(d(x, y)) d \mu(x)\right) g(y) d \mu(y) \\
& \leq \int_{B(z, 2 r)}\left(\int_{B(y, 4 r)} \rho_{\varepsilon}(d(x, y)) d \mu(x)\right) g(y) d \mu(y)
\end{aligned}
$$

by the Fubini theorem and a geometric observation. Since we are assuming (1.1), we can use a routine dyadic decomposition to obtain

$$
\int_{B(z, r)} J_{1}(x) d \mu(x) \leq \int_{B(z, 2 r)}\left(\sum_{j=0}^{\infty} \int_{B\left(y, 2^{-j+2} r\right) \backslash B\left(y, 2^{-j+1} r\right)} \rho_{\varepsilon}(d(x, y)) d \mu(x)\right) g(y) d \mu(y) .
$$

We now insert the definition of $\rho_{\varepsilon}$ (see (2.6) above) and we have

$$
\begin{aligned}
& \int_{B(z, r)} J_{1}(x) d \mu(x) \\
& \quad \leq \int_{B(z, 2 r)}\left(\sum_{j=0}^{\infty} \int_{B\left(y, 2^{-j+2} r\right) \backslash B\left(y, 2^{-j+1} r\right)} \frac{\left(\log \left(c+1 /\left(2^{-j+2} r\right)\right)\right)^{-\varepsilon-1}}{\mu\left(B\left(x, 2^{-j+4} r\right)\right)} d \mu(x)\right) g(y) d \mu(y) .
\end{aligned}
$$

A geometric observation shows that $\mu\left(B\left(x, 2^{-j+4} r\right)\right) \geq \mu\left(B\left(y, 2^{-j+2} r\right)\right)$ provided $d(x, y) \leq$ $2^{-j+2} r$. Thus, we have

$$
\int_{B(z, r)} J_{1}(x) d \mu(x) \leq \int_{B(z, 2 r)}\left(\sum_{j=0}^{\infty}\left(\log \left(c+1 /\left(2^{-j+2} r\right)\right)\right)^{-\varepsilon-1}\right) g(y) d \mu(y) .
$$

Now, we invoke the assumption that $\varepsilon>0$ and that $r<d_{G}$. From these conditions, (2.13) and the definition of $g$, we deduce

$$
\begin{aligned}
\int_{B(z, r)} J_{1}(x) d \mu(x) & \leq C(\log (c+1 / r))^{-\varepsilon} \int_{B(z, 2 r)} g(y) d \mu(y) \\
& \leq C r^{-v(z)}(\log (c+1 / r))^{-\beta(z)-\varepsilon} \mu(B(z, 4 r))
\end{aligned}
$$


so that

$$
\frac{1}{\mu(B(z, 4 r))} \int_{B(z, r)} J_{1}(x) d \mu(x) \leq C r^{-v(z)}(\log (c+1 / r))^{-\beta(z)-\varepsilon} .
$$

Next, we claim that $x \in B(z, r)$ and $y \notin B(z, 2 r)$ imply that

$$
\frac{2}{3} d(x, y) \leq d(y, z) \leq 2 d(x, y)
$$

and that

$$
B(x, 9 d(x, y)) \supset B(z, 4 d(z, y)) .
$$

Here we check only (2.19). When $w \in B(z, 4 d(z, y))$, we have

$$
d(w, x) \leq d(z, x)+d(w, z) \leq d(z, x)+4 d(z, y) \leq \frac{1}{2} d(y, z)+4 d(z, y)=\frac{9}{2} d(z, y) \leq 9 d(y, x) .
$$

We use (1.1), (2.18) and (2.19) for $J_{2}(x)$ to obtain

$$
\begin{aligned}
J_{2}(x) & =\int_{G \backslash B(z, 2 r)} \rho_{\varepsilon}(d(x, y)) g(y) d \mu(y) \\
& \leq C \int_{G \backslash B(z, 2 r)} \frac{(\log (c+1 / d(z, y)))^{-\varepsilon-1}}{\mu(B(x, 4 d(z, y)))} g(y) d \mu(y) \\
& =C \sum_{j=1}^{\infty} \int_{B\left(z, j^{j+1} r\right) \backslash B\left(z, 2^{\prime} r\right)} \frac{(\log (c+1 / d(z, y)))^{-\varepsilon-1}}{\mu(B(z, 4 d(z, y)))} g(y) d \mu(y) .
\end{aligned}
$$

Consequently, from (2.2), (2.13) and the assumption that $\beta$ is a bounded function, we obtain

$$
\begin{aligned}
J_{2}(x) & \leq C \sum_{j=1}^{\infty} \frac{\left(\log \left(c+1 /\left(2^{j+1} r\right)\right)\right)^{-\varepsilon-1}}{\mu\left(B\left(z, 2^{j+2} r\right)\right)} \int_{B\left(z, 2^{j+1} r\right)} g(y) d \mu(y) \\
& \leq C \sum_{j=1}^{\infty}\left(2^{j+1} r\right)^{-\nu(z)}\left(\log \left(c+1 /\left(2^{j+1} r\right)\right)\right)^{-\beta(z)-\varepsilon-1} .
\end{aligned}
$$

Recall that $v_{-}>0$ and that $\beta$ is bounded. Therefore, it follows that

$$
J_{2}(x) \leq C \int_{2 r}^{2 d_{G}} t^{-\nu(z)}(\log (c+1 / t))^{-\beta(z)-\varepsilon} \frac{d t}{t} \leq C r^{-\nu(z)}(\log (c+1 / r))^{-\beta(z)-\varepsilon}
$$

for $x \in B(z, r)$. Hence, we see that

$$
\frac{1}{\mu(B(z, 4 r))} \int_{B(z, r)} J_{2}(x) d \mu(x) \leq C r^{-v(z)}(\log (c+1 / r))^{-\beta(z)-\varepsilon} .
$$

From (2.16), (2.17) and (2.20), we conclude (2.15).

Now we are ready to prove Theorem 2.1. 
Proof of Theorem 2.1 We may assume that $f \geq 0$. For $\delta>0$, write

$$
U_{\alpha(\cdot), 9} f(x)=I_{1}(\delta)+I_{2}(\delta)
$$

according to (2.8). In view of Lemma 2.3, we find

$$
\begin{aligned}
I_{1}(\delta) \leq & C\left\{\delta^{\alpha(x)-v(x) / p(x)}\left(\log \left(c+\delta^{-1}\right)\right)^{-(q(x)+\beta(x)) / p(x)}\right. \\
& \left.+\delta^{\alpha(x)+(p(x)-1) v(x) / p(x)}\left(\log \left(c+\delta^{-1}\right)\right)^{\beta(x)-(q(x)+\beta(x)) / p(x)+(1+\varepsilon)} J\right\}
\end{aligned}
$$

with $J=J_{\varepsilon} f(x)$. Moreover, Lemma 2.4 yields

$$
I_{2}(\delta) \leq C \delta^{\alpha(x)-v(x) / p(x)}\left(\log \left(c+\delta^{-1}\right)\right)^{-(q(x)+\beta(x)) / p(x)},
$$

so that

$$
\begin{aligned}
U_{\alpha(\cdot), 9} f(x) \leq & C\left\{\delta^{\alpha(x)-v(x) / p(x)}\left(\log \left(c+\delta^{-1}\right)\right)^{-(q(x)+\beta(x)) / p(x)}\right. \\
& \left.+\delta^{\alpha(x)+(p(x)-1) v(x) / p(x)}\left(\log \left(c+\delta^{-1}\right)\right)^{\beta(x)-(q(x)+\beta(x)) / p(x)+(1+\varepsilon)} J\right\} .
\end{aligned}
$$

Now, letting

$$
\delta \equiv \min \left\{d_{G}, J^{-1 / v(x)}(\log (c+J))^{-(\beta(x)+(1+\varepsilon)) / v(x)}\right\}
$$

we obtain

$$
U_{\alpha(\cdot), 9} f(x) \leq C\left\{1+J^{1 / p^{*}(x)}(\log (c+J))^{-\alpha(x) \beta(x) / v(x)-q(x) / p(x)+(1+\varepsilon) / p^{*}(x)}\right\}
$$

By Lemma 2.5, we obtain

$$
\begin{aligned}
& \frac{1}{\mu(B(z, 4 r))} \int_{B(z, r)} \Psi\left(x, U_{\alpha(\cdot), 9} f(x)\right)\left(\log \left(c+U_{\alpha(\cdot), 9} f(x)\right)\right)^{-(1+\varepsilon)} d \mu(x) \\
& \quad \leq C \frac{1}{\mu(B(z, 4 r))} \int_{B(z, r)}(1+J) d \mu(x) \leq C r^{-v(z)}(\log (c+1 / r))^{-\beta(z)-\varepsilon}
\end{aligned}
$$

for $z \in G$ and $0<r<d_{G}$, which completes the proof of Theorem 2.1.

Remark 2.6 [14, Example 2.11] shows that Theorem 2.1 is best possible to exponents appearing in the Morrey condition.

\section{Sobolev's inequality in the case $p_{-}=1$ and $q_{-}>0$}

Let $p_{-}=1$ and $q_{-}>0$. In this section, we assume that there exists a constant $q_{0}>0$ such that

$$
s^{p(x)-1}(\log (c+s))^{q(x)-q_{0}} \leq C t^{p(x)-1}(\log (c+t))^{q(x)-q_{0}},
$$

whenever $0<s<t<\infty$ and $x \in X$. A direct consequence of (3.1) is that

$$
s^{p(x)-1}(\log (c+s))^{q(x)-\rho} \leq C t^{p(x)-1}(\log (c+t))^{q(x)-\rho}
$$

for $0<\rho \leq q_{0}$. Let $p^{*}$ and $\Psi$ be as in (2.4) and (2.5), respectively. Under this assumption, Theorem 2.1 is shown to be valid even for $\varepsilon=0$. 
Theorem 3.1 Let $p_{-}=1$ and $q_{-}>0$. Define $\Psi$ by (2.5). Assume that $p(\cdot), q(\cdot), \alpha(\cdot)$ and $v(\cdot)$ satisfy (2.1) and (3.1). Then there exists a constant $C>0$ such that

$$
\begin{aligned}
& \frac{1}{\mu(B(z, 4 r))} \int_{B(z, r)} \Psi\left(x, U_{\alpha(\cdot), 9} f(x)\right)\left(\log \left(c+U_{\alpha(\cdot), 9} f(x)\right)\right)^{-1} d \mu(x) \\
& \quad \leq C r^{-v(z)}(\log (c+1 / r))^{-\beta(z)}
\end{aligned}
$$

for all $z \in G$ and $0<r<d_{G}$, whenever $f$ is a nonnegative $\mu$-measurable function on $G$ satisfying $\|f\|_{L^{\Phi, v, \beta ; 2}(G)} \leq 1$.

For $\varepsilon>0$ and $x \in X$, we let

$$
\rho_{-\varepsilon}(r) \equiv \frac{1}{\mu(B(x, 9 r))}(\log (c+1 / r))^{\varepsilon-1}
$$

as before.

Note that this definition extends naturally (2.6). For a nonnegative $\mu$-measurable function $f$ on $G$, we define the (non-linear) logarithmic potential

$$
\begin{aligned}
L_{\varepsilon} f(x) \equiv & \int_{\left\{y \in G: d(x, y)^{-\varepsilon}<f(y)\right\}} \rho_{-\varepsilon}(d(x, y))(\log (c+f(y)))^{-\varepsilon} \\
& \times f(y)^{p(y)}(\log (c+f(y)))^{q(y)} d \mu(y) .
\end{aligned}
$$

To prove Theorem 3.1, we modify Lemmas 2.3 and 2.5 in the following manner, respectively.

Lemma 3.2 Let $0<\varepsilon \leq q_{0} / 2$ and define

$$
F(\delta) \equiv \int_{\left\{y \in B(x, \delta): d(x, y)^{-\varepsilon}<f(y)\right\}} \frac{d(x, y)^{\alpha(x)}}{\mu(B(x, 9 d(x, y)))}\left(\frac{\log (c+f(y))}{\log (c+1 / d(x, y))}\right)^{\varepsilon} f(y) d \mu(y)
$$

for $0<\delta<d_{G}$ and a nonnegative $\mu$-measurable function $f$ on $G$. Then there exists a constant $C>0$ such that

$$
\begin{aligned}
F(\delta) \leq & C\left\{\delta^{\alpha(x)-v(x) / p(x)}\left(\log \left(c+\delta^{-1}\right)\right)^{-(q(x)+\beta(x)) / p(x)}\right. \\
& \left.+\delta^{\alpha(x)+(p(x)-1) \nu(x) / p(x)}\left(\log \left(c+\delta^{-1}\right)\right)^{\beta(x)-(q(x)+\beta(x)) / p(x)+1} L_{\varepsilon} f(x)\right\} .
\end{aligned}
$$

Proof We abbreviate $\left\{y \in B(x, \delta): d(x, y)^{-\varepsilon}<f(y)\right\}$ to $E$. For a constant $k>0$, which will be specified in (3.6), we let

$$
E_{k}^{1} \equiv\left\{y \in B(x, \delta): d(x, y)^{-\varepsilon}<f(y) \leq k\right\}, \quad E_{k}^{2} \equiv E \backslash E_{k}^{1} .
$$

On $E_{k}^{1}$, using (1.1) and the dyadic decomposition, we estimate $f$ very crudely to obtain

$$
\begin{aligned}
& \int_{E_{k}^{1}} \frac{d(x, y)^{\alpha(x)}}{\mu(B(x, 9 d(x, y)))}\left(\frac{\log (c+f(y))}{\log (c+1 / d(x, y))}\right)^{\varepsilon} f(y) d \mu(y) \\
& \quad \leq k(\log (c+k))^{\varepsilon} \int_{B(x, \delta)} \frac{d(x, y)^{\alpha(x)}}{\mu(B(x, 9 d(x, y)))}(\log (c+1 / d(x, y)))^{-\varepsilon} d \mu(y)
\end{aligned}
$$




$$
\begin{aligned}
& =k(\log (c+k))^{\varepsilon} \sum_{j=1}^{\infty} \int_{B\left(x, 2^{-j+1} \delta\right) \backslash B\left(x, 2^{-j} \delta\right)} \frac{d(x, y)^{\alpha(x)}}{\mu(B(x, 9 d(x, y)))}(\log (c+1 / d(x, y)))^{-\varepsilon} d \mu(y) \\
& \leq k(\log (c+k))^{\varepsilon} \sum_{j=1}^{\infty} \int_{B\left(x, 2^{-j+1} \delta\right)} \frac{\left(2^{-j+1} \delta\right)^{\alpha(x)}}{\mu\left(B\left(x, 2^{-j+3} \delta\right)\right)}\left(\log \left(c+1 /\left(2^{-j+1} \delta\right)\right)\right)^{-\varepsilon} d \mu(y) \\
& \leq k(\log (c+k))^{\varepsilon} \sum_{j=1}^{\infty}\left(2^{-j+1} \delta\right)^{\alpha(x)}\left(\log \left(c+1 /\left(2^{-j+1} \delta\right)\right)\right)^{-\varepsilon} .
\end{aligned}
$$

If we pass to a continuous variable and keep in mind that $\alpha_{-}>0$, then we obtain

$$
\begin{aligned}
& \int_{E_{k}^{1}} \frac{d(x, y)^{\alpha(x)}}{\mu(B(x, 9 d(x, y)))}\left(\frac{\log (c+f(y))}{\log (c+1 / d(x, y))}\right)^{\varepsilon} f(y) d \mu(y) \\
& \quad \leq C k(\log (c+k))^{\varepsilon} \int_{0}^{\delta} t^{\alpha(x)-1}(\log (c+1 / t))^{-\varepsilon} d t \\
& \quad \leq C k(\log (c+k))^{\varepsilon} \delta^{\alpha(x)-\alpha_{-} / 2}\left(\log \left(c+\delta^{-1}\right)\right)^{-\varepsilon} \int_{0}^{\delta} t^{\alpha_{-} / 2-1} d t \\
& \quad=C k(\log (c+k))^{\varepsilon} \delta^{\alpha(x)}\left(\log \left(c+\delta^{-1}\right)\right)^{-\varepsilon} .
\end{aligned}
$$

In summary, we obtained

$$
\begin{aligned}
& \int_{E_{k}^{1}} \frac{d(x, y)^{\alpha(x)}}{\mu(B(x, 9 d(x, y)))}\left(\frac{\log (c+f(y))}{\log (c+1 / d(x, y))}\right)^{\varepsilon} f(y) d \mu(y) \\
& \quad \leq C k(\log (c+k))^{\varepsilon} \delta^{\alpha(x)}\left(\log \left(c+\delta^{-1}\right)\right)^{-\varepsilon} .
\end{aligned}
$$

On $E_{k}^{2}$, we use (3.2) and $\varepsilon \leq q_{0} / 2$.

$$
\begin{aligned}
& \int_{E_{k}^{2}} \frac{d(x, y)^{\alpha(x)}}{\mu(B(x, 9 d(x, y)))}\left(\frac{\log (c+f(y))}{\log (c+1 / d(x, y))}\right)^{\varepsilon} f(y) d \mu(y) \\
& \leq C \int_{E_{k}^{2}} \frac{d(x, y)^{\alpha(x)}}{\mu(B(x, 9 d(x, y)))}\left(\frac{\log (c+f(y))}{\log (c+1 / d(x, y))}\right)^{\varepsilon} f(y) \\
& \quad \times\left(\frac{f(y)}{k}\right)^{p(y)-1}\left(\frac{\log (c+f(y))}{\log (c+k)}\right)^{q(y)-2 \varepsilon} d \mu(y) \\
&=C \int_{E_{k}^{2}} \frac{d(x, y)^{\alpha(x)}(\log (c+1 / d(x, y)))^{-\varepsilon}(\log (c+f(y)))^{-\varepsilon}}{\mu(B(x, 9 d(x, y)))} g(y) \frac{k^{1-p(y)}}{(\log (c+k))^{q(y)-2 \varepsilon}} d \mu(y) \\
& \leq C \delta^{\alpha(x)}\left(\log \left(c+\delta^{-1}\right)\right)^{1-2 \varepsilon} \\
& \quad \times \int_{E} \rho_{-\varepsilon}(d(x, y))(\log (c+f(y)))^{-\varepsilon} g(y) \frac{k^{1-p(y)}}{(\log (c+k))^{q(y)-2 \varepsilon}} d \mu(y)
\end{aligned}
$$

for all $x \in G$, where $g(y)=f(y)^{p(y)}(\log (c+f(y)))^{q(y)}$. Hence, from (3.5), we deduce

$$
\begin{aligned}
F(\delta) \leq & C\left\{k(\log (c+k))^{\varepsilon} \delta^{\alpha(x)}\left(\log \left(c+\delta^{-1}\right)\right)^{-\varepsilon}+\delta^{\alpha(x)}\left(\log \left(c+\delta^{-1}\right)\right)^{1-2 \varepsilon}\right. \\
& \left.\times \int_{E} \rho_{-\varepsilon}(d(x, y))(\log (c+f(y)))^{-\varepsilon} g(y) \frac{k^{1-p(y)}}{(\log (c+k))^{q(y)-2 \varepsilon}} d \mu(y)\right\} .
\end{aligned}
$$


We set

$$
k \equiv \delta^{-v(x) / p(x)}\left(\log \left(c+\delta^{-1}\right)\right)^{-(q(x)+\beta(x)) / p(x)} .
$$

Then we have for $y \in B(x, \delta)$,

$$
k^{-p(y)} \leq C k^{-p(x)}
$$

and

$$
(\log (c+k))^{-q(y)} \leq C(\log (c+k))^{-q(x)}
$$

as we did in (2.11) and (2.12). Consequently, it follows that

$$
\begin{aligned}
F(\delta) \leq & C\left\{\delta^{\alpha(x)-v(x) / p(x)}\left(\log \left(c+\delta^{-1}\right)\right)^{-(q(x)+\beta(x)) / p(x)}\right. \\
& \left.+\delta^{\alpha(x)+(p(x)-1) v(x) / p(x)}\left(\log \left(c+\delta^{-1}\right)\right)^{\beta(x)-(q(x)+\beta(x)) / p(x)+1} L_{\varepsilon} f(x)\right\} .
\end{aligned}
$$

Now the result follows.

The next lemma is a counterpart for Lemma 2.5 .

Lemma 3.3 Let $f$ be a nonnegative $\mu$-measurable function satisfying $\|f\|_{L^{\Phi, v, \beta ; 2}(G)} \leq 1$ and define $L_{\varepsilon} f(x)$ by (3.4). If $\Phi$ is given by (1.5), then there exists a constant $C>0$ independent of $z \in G, 0<r<d_{G}$ and $f$ such that

$$
\frac{1}{\mu(B(z, 4 r))} \int_{B(z, r)} L_{\varepsilon} f(x) d \mu(x) \leq C r^{-v(z)}(\log (c+1 / r))^{-\beta(z)} .
$$

Proof Let $f$ be a nonnegative $\mu$-measurable function on $G$ satisfying $\|f\|_{L^{\Phi, v, \beta ; 2}(G)} \leq 1$. Write

$$
\begin{aligned}
L_{\varepsilon} f(x)= & \int_{\left\{y \in B(z, 2 r): d(x, y)^{-\varepsilon}<f(y)\right\}} \rho_{-\varepsilon}(d(x, y))(\log (c+f(y)))^{-\varepsilon} g(y) d \mu(y) \\
& +\int_{\left\{y \in G \backslash B(z, 2 r): d(x, y)^{-\varepsilon}<f(y)\right\}} \rho_{-\varepsilon}(d(x, y))(\log (c+f(y)))^{-\varepsilon} g(y) d \mu(y) \\
= & L_{1}(x)+L_{2}(x),
\end{aligned}
$$

where $g(y) \equiv f(y)^{p(y)}(\log (c+f(y)))^{q(y)}$. Let us estimate

$$
\begin{aligned}
& \int_{B(z, r)} L_{1}(x) d \mu(x) \\
& \quad=\int_{B(z, r)}\left(\int_{\left\{x \in G: d(x, y)^{-\varepsilon}<f(y)\right\}} \rho_{-\varepsilon}(d(x, y)) d \mu(x)\right)(\log (c+f(y)))^{-\varepsilon} g(y) d \mu(y) .
\end{aligned}
$$

For each $y \in G$, we decompose

$$
\left\{x \in G: d(x, y)^{-\varepsilon}<f(y)\right\}=\bigcup_{j=0}^{\infty}\left(B\left(y, 2^{j+1} f(y)^{-1 / \varepsilon}\right) \backslash B\left(y, 2^{j} f(y)^{-1 / \varepsilon}\right)\right) .
$$


Then, using (3.3), (3.9) and a crude estimate of the integrand, we obtain

$$
\begin{aligned}
& \int_{B(z, r)} L_{1}(x) d \mu(x) \\
& \leq C \int_{B(z, 2 r)}\left(\sum_{j=0}^{\infty} \int_{B\left(y, j^{j+1} f(y)^{-1 / \varepsilon}\right)} \frac{\left(\log \left(c+1 /\left(2^{j} f(y)^{-1 / \varepsilon}\right)\right)\right)^{\varepsilon-1}}{\mu\left(B\left(x, 2^{j+3} f(y)^{-1 / \varepsilon}\right)\right)} d \mu(x)\right) \\
& \quad \times(\log (c+f(y)))^{-\varepsilon} g(y) d \mu(y) \\
& \leq C \int_{B(z, 2 r)}\left(\sum_{j=0}^{\infty}\left(\log \left(c+1 /\left(2^{j} f(y)^{-1 / \varepsilon}\right)\right)\right)^{\varepsilon-1}\right)(\log (c+f(y)))^{-\varepsilon} g(y) d \mu(y) .
\end{aligned}
$$

From this and the fact that $\|f\|_{L^{\Phi, v, \beta ; 2}(G)} \leq 1$, we deduce

$$
\int_{B(z, r)} L_{1}(x) d \mu(x) \leq C \int_{B(z, 2 r)} g(y) d \mu(y) \leq C r^{-v(z)}(\log (c+1 / r))^{-\beta(z)} \mu(B(z, 4 r)),
$$

so that

$$
\frac{1}{\mu(B(z, 4 r))} \int_{B(z, r)} L_{1}(x) d \mu(x) \leq C r^{-\nu(z)}(\log (c+1 / r))^{-\beta(z)} .
$$

Note that $x \in B(z, r)$ and $y \notin B(z, 2 r)$ imply that

$$
\frac{2}{3} d(x, y) \leq d(y, z) \leq 2 d(x, y)
$$

and that

$$
B(x, 9 d(x, y)) \supset B(z, 4 d(z, y)) .
$$

For $L_{2}$, we insert (3.3) directly to obtain a pointwise estimate for $x \in B(z, r)$ :

$$
\begin{aligned}
L_{2}(x) & \leq C \int_{G \backslash B(z, 2 r)} \frac{(\log (c+1 / d(x, y)))^{-1}}{\mu(B(x, 9 d(x, y)))} g(y) d \mu(y) \\
& \leq C \int_{G \backslash B(z, 2 r)} \frac{(\log (c+1 / d(z, y)))^{-1}}{\mu(B(z, 4 d(z, y)))} g(y) d \mu(y) .
\end{aligned}
$$

As before, we decompose $G \backslash B(z, 2 r)$ dyadically to estimate $L_{2}(x)$ from above. The result is

$$
\begin{aligned}
L_{2}(x) & \leq C \sum_{j=1}^{\infty} \int_{B\left(z, 2^{j+1} r\right) \backslash B\left(z, 2^{j} r\right)} \frac{(\log (c+1 / d(z, y)))^{-1}}{\mu(B(z, 4 d(z, y)))} g(y) d \mu(y) \\
& \leq C \sum_{j=1}^{\infty} \int_{B\left(z, 2^{j+1} r\right) \backslash B\left(z, 2^{j} r\right)} \frac{\left(\log \left(c+1 /\left(2^{j+1} r\right)\right)\right)^{-1}}{\mu\left(B\left(z, 2^{j+2} r\right)\right)} g(y) d \mu(y) \\
& \leq C \sum_{j=1}^{\infty} \frac{\left(\log \left(c+1 /\left(2^{j+1} r\right)\right)\right)^{-1}}{\mu\left(B\left(z, 2^{j+2} r\right)\right)} \int_{B\left(z, 2^{j+1} r\right)} g(y) d \mu(y) .
\end{aligned}
$$


From this and the fact that $\|f\|_{L^{\Phi, v, \beta ; 2}(G)} \leq 1$, we deduce

$$
\begin{aligned}
L_{2}(x) & \leq C \sum_{j=1}^{\infty}\left(2^{j+1} r\right)^{-v(z)}\left(\log \left(c+1 /\left(2^{j+1} r\right)\right)\right)^{-\beta(z)-1} \\
& \leq C \int_{2 r}^{2 d_{G}} t^{-v(z)}(\log (c+1 / t))^{-\beta(z)-1} \frac{d t}{t} \\
& \leq C r^{-v(z)}(\log (c+1 / r))^{-\beta(z)-1}
\end{aligned}
$$

for $x \in B(z, r)$. Hence, we see that

$$
\frac{1}{\mu(B(z, 4 r))} \int_{B(z, r)} L_{2}(x) d \mu(x) \leq C r^{-v(z)}(\log (c+1 / r))^{-\beta(z)-1}
$$

Thus, putting (3.8), (3.10) and (3.11) together, we obtain (3.7).

Proof of Theorem 3.1 We may assume that $f \geq 0$ by considering $|f|$ if necessary. Unlike Theorem 2.1, we have freedom to choose $\varepsilon$, so that we define $\varepsilon \equiv \min \left\{\alpha_{-} / 2, q_{0} / 2\right\}$. Let us obtain a pointwise estimate of $U_{\alpha(\cdot), 9} f(x)$ for $x \in G$.

For $\delta>0$, where $\delta$ is specified in (3.13) below, we decompose

$$
\begin{aligned}
U_{\alpha(\cdot), 9} f(x)= & \int_{B(x, \delta)} \frac{d(x, y)^{\alpha(x)} f(y)}{\mu(B(x, 9 d(x, y)))} d \mu(y) \\
& +\int_{G \backslash B(x, \delta)} \frac{d(x, y)^{\alpha(x)} f(y)}{\mu(B(x, 9 d(x, y)))} d \mu(y) \\
= & I_{1}(\delta)+I_{2}(\delta) .
\end{aligned}
$$

As in the proof of (2.9), we have

$$
\int_{B(x, \delta)} \frac{d(x, y)^{\alpha(x)-\varepsilon}}{\mu(B(x, 9 d(x, y)))} d \mu(y) \leq C \delta^{\alpha(x)-\varepsilon} \leq C
$$

since $\alpha_{-}-\varepsilon=\alpha_{-}-\min \left\{\alpha_{-} / 2, q_{0} / 2\right\}=\max \left\{\alpha_{-} / 2, \alpha_{-}-q_{0} / 2\right\}>0$.

Note that $\varepsilon \leq q_{0} / 2$. In view of Lemma 3.2, we find

$$
\begin{aligned}
I_{1}(\delta) \leq & \int_{B(x, \delta)} \frac{d(x, y)^{\alpha(x)-\varepsilon}}{\mu(B(x, 9 d(x, y)))} d \mu(y) \\
& +\int_{\left\{y \in B(x, \delta): d(x, y)^{-\varepsilon}<f(y)\right\}} \frac{d(x, y)^{\alpha(x)} f(y)}{\mu(B(x, 9 d(x, y)))}\left(\frac{(\log (c+f(y)))}{\log \left(c+d(x, y)^{-\varepsilon}\right)}\right)^{\varepsilon} f(y) d \mu(y) \\
\leq & C\left\{\delta^{\alpha(x)-v(x) / p(x)}\left(\log \left(c+\delta^{-1}\right)\right)^{-(q(x)+\beta(x)) / p(x)}\right. \\
& \left.+\delta^{\alpha(x)+(p(x)-1) v(x) / p(x)}\left(\log \left(c+\delta^{-1}\right)\right)^{\beta(x)-(q(x)+\beta(x)) / p(x)+1} L\right\}
\end{aligned}
$$

with $L=L_{\varepsilon} f(x)$. Moreover, Lemma 2.4 yields

$$
I_{2}(\delta) \leq C \delta^{\alpha(x)-v(x) / p(x)}\left(\log \left(c+\delta^{-1}\right)\right)^{-(q(x)+\beta(x)) / p(x)},
$$


so that

$$
\begin{aligned}
U_{\alpha(\cdot), 9} f(x) \leq & C\left\{\delta^{\alpha(x)-v(x) / p(x)}\left(\log \left(c+\delta^{-1}\right)\right)^{-(q(x)+\beta(x)) / p(x)}\right. \\
& \left.+\delta^{\alpha(x)+(p(x)-1) v(x) / p(x)}\left(\log \left(c+\delta^{-1}\right)\right)^{\beta(x)-(q(x)+\beta(x)) / p(x)+1} L\right\} .
\end{aligned}
$$

Now, letting

$$
\delta \equiv \min \left\{d_{G}, L^{-1 / v(x)}(\log (c+L))^{-(\beta(x)+1) / v(x)}\right\}
$$

we obtain

$$
U_{\alpha(\cdot), 9} f(x) \leq C\left\{1+L^{1 / p^{*}(x)}(\log (c+L))^{-\alpha(x) \beta(x) / v(x)-q(x) / p(x)+1 / p^{*}(x)}\right\} .
$$

In view of Lemma 3.3, we find

$$
\begin{aligned}
& \frac{1}{\mu(B(z, 4 r))} \int_{B(z, r)} \Psi\left(x, U_{\alpha(\cdot), 9} f(x)\right)\left(\log \left(c+U_{\alpha(\cdot), 9} f(x)\right)\right)^{-1} d \mu(x) \\
& \leq C \frac{1}{\mu(B(z, 4 r))} \int_{B(z, r)}(1+L) d \mu(x) \leq C r^{-v(z)}(\log (c+1 / r))^{-\beta(z)},
\end{aligned}
$$

which completes the proof of Theorem 3.1.

Remark 3.4 [14, Example 3.6] shows that Theorem 3.1 is best possible to exponents appearing in the Morrey condition.

\section{Competing interests}

The authors declare that they have no competing interests.

\section{Authors' contributions}

All the authors participated in all the discussions and they read and approved the final manuscript.

\section{Author details}

${ }^{1}$ Department of Mathematics, Kyoto University, Kyoto, 606-8502, Japan. ${ }^{2}$ Current address: Department of Mathematics and Information Sciences, Tokyo Metropolitan University, Minami-Ohsawa 1-1, Hachioji-shi, Tokyo 192-0397, Japan.

${ }^{3}$ Department of Mathematics Graduate School of Education, Hiroshima University, Higashi-Hiroshima, 739-8524, Japan.

\section{Acknowledgements}

The authors are thankful to the anonymous referees for their valuable comments about the history of fractional integral operators on non-doubling measure spaces.

Received: 18 June 2012 Accepted: 12 November 2012 Published: 7 January 2013

\section{References}

1. Morrey, CB: On the solutions of quasi-linear elliptic partial differential equations. Trans. Am. Math. Soc. 43, 126-166 (1938)

2. Peetre, J: On the theory of $L_{p, \lambda}$ spaces. J. Funct. Anal. 4, 71-87 (1969)

3. Kokilashvili, V: Weighted estimates for classical integral operators. In: Nonlinear Analysis, Function Spaces and Appl. IV, pp. 86-103. Teubner, Leipzig (1976)

4. Edmunds, D, Kokilashvili, V, Meskhi, A: Bounded and Compact Integral Operators. Kluwer Academic, Dordrecht (2002)

5. Adams, DR: A note on Riesz potentials. Duke Math. J. 42, 765-778 (1975)

6. Sawano, Y, Sugano, S, Tanaka, H: Orlicz-Morrey spaces and fractional operators. Potential Anal. 36(4), $517-556$ (2012)

7. Nazarov, F, Treil, S, Volberg, A: Cauchy integral and Calderón-Zygmund operators on nonhomogeneous spaces. Int. Math. Res. Not. 1997(15), 703-726 (1997)

8. Nazarov, F, Treil, S, Volberg, A: Weak type estimates and Cotlar inequalities for Calderön-Zygmund operators on nonhomogeneous spaces. Int. Math. Res. Not. 1998, 463-487 (1998)

9. Sawano, Y, Shimomura, T: Sobolev embeddings for Riesz potentials of functions in non-doubling Morrey spaces of variable exponents. Collect. Math. (to appear) 
10. Futamura, T, Mizuta, Y, Shimomura, T: Sobolev embedding for variable exponent Riesz potentials on metric spaces. Ann. Acad. Sci. Fenn. Math. 31, 495-522 (2006)

11. Kokilashvili, V, Meskhi, A: Maximal functions and potentials in variable exponent Morrey spaces with non-doubling measure. Complex Var. Elliptic Equ. 55(8-10), 923-936 (2010)

12. Mizuta, Y, Nakai, E, Ohno, T, Shimomura, T: An elementary proof of Sobolev embeddings for Riesz potentials of functions in Morrey spaces $L^{1, v, \beta}(G)$. Hiroshima Math. J. 38, 425-436 (2008)

13. Mizuta, Y, Nakai, E, Ohno, T, Shimomura, T: Boundedness of fractional integral operators on Morrey spaces and Sobolev embeddings for generalized Riesz potentials. J. Math. Soc. Jpn. 62, 707-744 (2010)

14. Mizuta, Y, Nakai, E, Ohno, T, Shimomura, T: Riesz potentials and Sobolev embeddings on Morrey spaces of variable exponent. Complex Var. Elliptic Equ. 56(7-9), 671-695 (2011)

15. Mizuta, Y, Shimomura, T: Sobolev embeddings for Riesz potentials of functions in Morrey spaces of variable exponent. J. Math. Soc. Jpn. 60, 583-602 (2008)

16. Mizuta, Y, Shimomura, T, Sobukawa, T: Sobolev's inequality for Riesz potentials of functions in non-doubling Morrey spaces. Osaka J. Math. 46, 255-271 (2009)

17. Sawano, Y, Tanaka, H: Morrey spaces for non-doubling measures. Acta Math. Sin. Engl. Ser. 21, 1535-1544 (2005)

18. Maeda, F-Y, Mizuta, Y, Ohno, T: Approximate identities and Young type inequalities in variable Lebesgue-Orlicz spaces $L^{p(\cdot)}(\log L)^{q(\cdot)}$. Ann. Acad. Sci. Fenn. Math. 35, 405-420 (2010)

19. Gunawan, H, Sawano, Y, Sihwaningrum, I: Fractional integral operators in nonhomogeneous spaces. Bull. Aust. Math. Soc. 80(2), 324-334 (2009)

20. Sawano, Y: Generalized Morrey spaces for non-doubling measures. Nonlinear Differ. Equ. Appl. 15(4-5), 413-425 (2008)

21. Sawano, Y, Wadade, H: On the Gagliardo-Nirenberg type inequality in the critical Sobolev-Morrey space, J. Fourier Anal. Appl., online

22. Stein, EM: Singular Integrals and Differentiability Properties of Functions. Princeton Univ. Press, Princeton (1970)

23. Hedberg, LI: On certain convolution inequalities. Proc. Am. Math. Soc. 36, 505-510 (1972)

24. Sawano, Y: Sharp estimates of the modified Hardy-Littlewood maximal operator on the nonhomogeneous space via covering lemmas. Hokkaido Math. J. 34, 435-458 (2005)

25. Terasawa, Y: Outer measures and weak type $(1,1)$ estimates of Hardy-Littlewood maximal operators. J. Inequal. Appl. 2006, Art. ID 15063 (2006)

26. Mizuta, Y, Ohno, T, Shimomura, T: Sobolev embeddings for Riesz potential spaces of variable exponents near 1 and Sobolev's exponent. Bull. Sci. Math. 134, 12-36 (2010)

27. Nakai, E: Generalized fractional integrals on Orlicz-Morrey spaces. In: Banach and Function Spaces, pp. 323-333. Yokohama Publ., Yokohama (2004)

28. O’Neil, R: Fractional integration in Orlicz spaces. I. Trans. Am. Math. Soc. 115, 300-328 (1965)

doi:10.1186/1029-242X-2013-12

Cite this article as: Sawano and Shimomura: Sobolev's inequality for Riesz potentials of functions in generalized Morrey spaces with variable exponent attaining the value 1 over non-doubling measure spaces. Journal of Inequalities and Applications 2013 2013:12.

\section{Submit your manuscript to a SpringerOpen ${ }^{\circ}$ journal and benefit from:}

- Convenient online submission

Rigorous peer review

- Immediate publication on acceptance

- Open access: articles freely available online

- High visibility within the field

- Retaining the copyright to your article 